\title{
Discovery of a new cataclysmic variable through optical variability and X-ray emission
}

\author{
M. L. Pretorius and C. Knigge \\ School of Physics and Astronomy, University of Southampton, Highfield, Southampton SO17 1BJ, UK \\ e-mail: [mlp; christian]@astro.soton.ac.uk \\ Received 19 September 2006 / Accepted 16 October 2006 \\ ABSTRACT

\begin{abstract}
Aims. We present discovery observations of the new cataclysmic variable star (CV) 1RXS J092737.4-191529, as well as spectra and novae with high outburst duty cycles. targets for follow-up observations. Long-slit spectra were taken to identify CVs in the sample.

Results. Two out of three objects selected in this way are CVs. One of these is the known dwarf nova SY Vol, while the second system, 1RXS J092737.4-191529, is a new discovery. We present medium resolution spectra, UBVRI magnitudes, and high-speed photometry for both these CVs. Rapid flickering in the light curve of 1RXS J092737.4-191529 confirms the mass transferring binary
\end{abstract} \\ photometry of SY Vol. The selection technique that turned up these two CVs is described; it should be efficient for finding dwarf \\ Methods. Two very common observational features of $\mathrm{CV}$ s, namely optical variability and X-ray emission, are combined to select \\ nature of this object; it is probably a dwarf nova that was in quiescence during our observations.
}

Key words. stars: dwarf novae - stars: novae, cataclysmic variables - stars: variables: general

\section{Introduction}

Cataclysmic variable stars (CVs) are semi-detached binary stars, consisting of a white dwarf primary accreting from a companion that is usually a late-type, approximately main-sequence star. The orbital periods $\left(P_{\text {orb }}\right)$ of these binaries are typically on the order of hours. Warner (1995) gives a comprehensive review of the subject.

Based on their long term photometric behaviour, nonmagnetic CVs are divided into the classes dwarf novae (DNe) and nova-like variables (NLs). The physical distinction between them is that the time-averaged mass transfer rate $(\dot{M})$ is lower in DNe than in NLs. The majority of CVs are DNe; the defining characteristic of this class is outbursts during which the system brightens by typically 2-5 mag. Outburst durations are days to tens of days, and the recurrence interval ranges from tens of days to decades. NLs are usually in a state of high $\dot{M}$, but a subtype of NLs, called VY Scl stars, are characterised by occasional low states (at least 1 mag fainter than the average brightness, lasting for weeks to years; see Wade \& Ward 1985). DN outburst are caused by an accretion disc instability (e.g. Osaki 1996), while VY Scl star low states probably result from a reduction in the rate at which the secondary star loses mass (e.g. Livio \& Pringle 1994).

Many magnetic CVs (see Cropper 1990; Patterson 1994, for reviews) vary by several magnitudes. In the case of polars, this is probably caused by the same mechanism as in VY Scl stars, while the high states seen in some intermediate polars (IPs) are (in at least some cases) believed to be caused by the disc instability that operates in DNe (Kim et al. 1992; Warner 1996; Hellier et al. 1997).

In addition to the mechanisms mentioned above, large amplitude variability in CVs is caused by nova eruptions, which occur in all types of hydrogen-rich CVs. All CVs are therefore expected to be large amplitude variables, although the recurrence times and duty cycles of the variability range widely.

A second attribute that should be shared by all active CVs is $\mathrm{X}$-ray emission generated in the accretion flow. X-ray observations of CVs indicate $10^{29} \mathrm{erg} \mathrm{s}^{-1} \lesssim L_{\mathrm{x}} \lesssim 3 \times 10^{32} \mathrm{erg} \mathrm{s}^{-1}$ (e.g. Patterson \& Raymond 1985; van Teeseling et al. 1996; Verbunt et al. 1997; Wheatley et al. 2000).

Given that large amplitude variability and X-ray emission are ubiquitous features of CVs, a combination of these two criteria is a promising way to identify CVs. Wide field photographic sky surveys have recorded large amplitude variability in many stars; the nature of the vast majority of these variables is not known. Furthermore, optical identifications exist for only a small fraction of all sources detected in the ROSAT All-Sky Survey (the RASS; see Voges et al. 1999, 2000). There should be many CVs amongst the unidentified sources in this survey. More than $100 \mathrm{CVs}$ have already been discovered by ROSAT (Gänsicke 2005). Optical follow-up of a sample of bright ( $>0.2$ counts $/ \mathrm{s})$ ROSAT sources led to the discovery of $11 \mathrm{CVs}$ (Schwope et al. 2002). In a search aimed specifically at CVs, Gänsicke et al. (2005) use a fainter ROSAT flux limit together with the requirements $J-K<1, K>11$, and $R<17$.

Here, we investigate the feasibility of selecting CVs for large amplitude optical variability and X-ray emission. We present our selection procedure and observations in the next two sections, before discussing and summarising the results in Sect. 4 .

\section{Selection}

Our selection is aimed at X-ray sources that can be associated with optical variables. We use two epochs of photographic images to identify large amplitude optical variables, and correlate these objects with sources detected in the RASS. Since we use only two observations to search for variability, we expect to 
Table 1. Log of the spectroscopic observations. The fourth column gives the photographic $R$ magnitude of the fainter of the two epochs, as well as the difference between the two $R$-band measurements. The dates are for the start of the night; coordinates are for the optical variables.

\begin{tabular}{|c|c|c|c|c|c|c|}
\hline Object & $\alpha_{2000}$ & $\delta_{2000}$ & $R(\Delta R)$ & Date & HJD 2453000.0+ & $t_{\text {int }} / \mathrm{s}$ \\
\hline 1RXS J085325.4-711255 & $08: 53: 26.2$ & $-71: 12: 48$ & $17.0(2.4)$ & 5 Jan. 2004 & 376.47567 & 1400 \\
\hline & & & & & & 1800 \\
\hline 1RXS J092737.4-191529 & $09: 27: 37.1$ & $-19: 15: 34$ & $18.3(2.9)$ & 004 & 463 & 1600 \\
\hline 1RXS J152912.9-101623 & $15: 29: 12.2$ & $-10: 16: 28$ & $18.4(2.0)$ & $\begin{array}{l}10 \text { Jan. } 2004 \\
4 \text { Mar. } 2006\end{array}$ & $\begin{array}{l}381.57730 \\
799.55906\end{array}$ & $\begin{array}{l}2000 \\
1300\end{array}$ \\
\hline
\end{tabular}

Notes: ${ }^{a}$ This is $\mathrm{SY} \mathrm{Vol;} t_{\text {int }}$ is the integration time.

find mainly objects with frequent, high duty cycle brightness variations.

The photographic plate digitising machine, SuperCOSMOS, has scanned Schmidt sky survey plates taken with the UK, European Southern Observatory (ESO), and Palomar Schmidt telescopes. The resulting data are publicly available and comprise the SuperCOSMOS Sky Survey (the SSS; see Hambly et al. 2001a-c). The survey covers the southern celestial hemisphere $(\delta<+3.0)$ at two epochs in $R$, as well as at a single epoch in $B$ and $I$. The data can be accessed most readily through the SuperCOSMOS Science Archive (Hambly et al. 2004).

Considering only a subset ${ }^{1}$ of sources detected in the SSS that consists mainly of genuine stars (rather than extended objects or plate defects) we choose objects that varied by more than 2 mag between the two $R$-band observations. In addition, we require $R<19$ in both epochs. This means that it should be possible to obtain follow-up observations with a 2-m class telescope, and excludes stars with high proper motion (without this requirement, many high proper motion objects are included, since they are detected in only one epoch).

A total of 2757 targets results from this selection. We inspected scans of both $R$-band plates for 100 of these objects - in only two out of these 100 cases is the variability clearly spurious, with obvious defects on the plates very near the stellar images. However, we have found that even apparent differences of $\gtrsim 2$ mag are occasionally caused by poor calibration between the different plates. Only 36 of the 2757 objects selected in this way can be matched to known variable stars in the SIMBAD Astronomical Database. This underlines the fact that a large number of stars that show large amplitude variability remain completely unstudied. Amongst the known variable stars in this sample are $8 \mathrm{CVs}^{2}, 13$ Mira variables, and 3 young stellar objects. Also included in the sample of variables are two quasars; both were detected by ROSAT.

Three of the optical variables found in the SSS are close positional matches to ROSAT sources that have not been optically identified; this includes the known CV SY Vol, which is very likely the optical counterpart of 1RXS J085325.4-711255. These three objects are selected for the present study.

1 This is the so-called ReliableStars subset, which consists of sources with small ellipticity, detected on at least 3 out of the 4 plates. Objects that were assigned quality flags indicating a possibly bad image during the image analysis are excluded, together with objects that were deblended. The ReliableStars are further restricted to areas away from the galactic plane and galactic bulge $\left(|b|>10^{\circ}\right.$ and $>20^{\circ}$ from the Galactic Centre), to avoid severely crowded fields.

2 They are WX Hyi, BI Ori, SY Vol, TU Ind, GS Pav, V803 Cen, SDSS J040714.78-064425.2, and SDSS J161332.56-000331.0. The two systems WX Hyi and V803 Cen are known to be the optical counterparts of RASS sources (Verbunt et al. 1997).

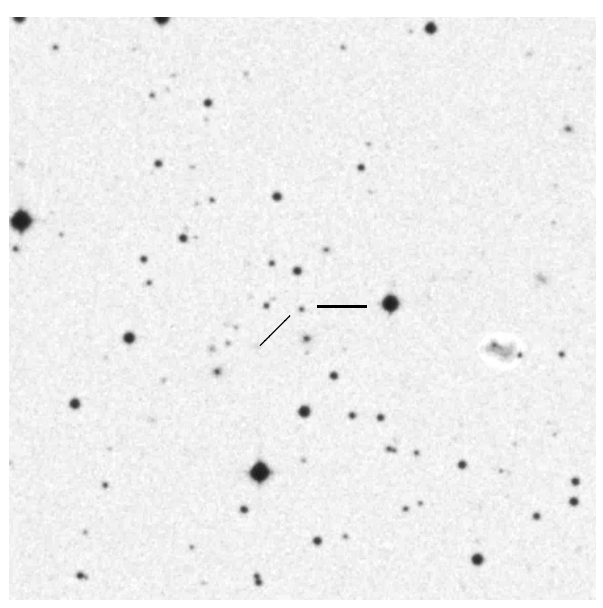

Fig. 1. A $5^{\prime} \times 5^{\prime}$ finding chart of the optical counterpart of 1RXS J092737.4-191529. This was made using a digitised ESO Schmidt telescope $R$-band plate. North is at the top and east to the left of the figure. The system is near minimum light in this image.

\section{Observations}

We obtained follow-up observations at the Sutherland site of the South African Astronomical Observatory (SAAO). In addition to SY Vol, 1RXS J092737.4-191529 proved to be a CV. A finding chart for this newly discovered CV is displayed in Fig. 1.

Identification spectra were taken with the Grating Spectrograph on the SAAO 1.9-m telescope. The spectrograph is equipped with an SITe charge-coupled device (CCD) detector, and was used with grating No. 7 and a slit width of 1."8, yielding a resolution of $\simeq 5 \AA$ over a range of $\simeq 3550 \AA$. Table 1 gives a $\log$ of the spectroscopic observations, together with positions and photographic $R$-band magnitudes of the targets.

The variable star that we associate with 1RXS J152912.9101623 was fainter than $R \simeq 19.0$ when we observed it, and close to the magnitude limit of the spectrograph. The $\mathrm{S} / \mathrm{N}$ in the spectrum is too low to allow for a reliable determination of the spectral type, but, since no emission lines were detected, it is unlikely that this object is a CV.

In addition to the identification spectroscopy, we obtained UBVRI photometry as well as high speed photometry of SY Vol and 1RXS J092737.4-191529, using the University of Cape Town CCD photometer (the UCT CCD; see O'Donoghue 1995) on the SAAO 1-m telescope. The UBVRI magnitudes of the CVs are listed in Table 2. Each of these measurements is the average of three exposures separated by a few minutes; Table 2 gives the photometric errors, but we emphasise that these are smaller than the amplitude of flickering (at least in white light). The $U-B$ and $B-V$ colours of both systems are well within the range observed in quiescent DNe (Bruch \& Engel 1994). Table 3 gives a $\log$ of the time-resolved photometry. These observations 
Table 2. UBVRI magnitudes of SY Vol and 1RXS J092737.4-191529.

\begin{tabular}{lll}
\hline \hline & SY Vol & 1RXS J092737.4-191529 \\
\hline$U$ & $17.66 \pm 0.06$ & $18.05 \pm 0.08$ \\
$B$ & $18.46 \pm 0.05$ & $18.96 \pm 0.06$ \\
$V$ & $18.08 \pm 0.02$ & $18.60 \pm 0.04$ \\
$R$ & $17.60 \pm 0.02$ & $18.00 \pm 0.03$ \\
$I$ & $16.88 \pm 0.01$ & $17.24 \pm 0.02$ \\
\hline
\end{tabular}

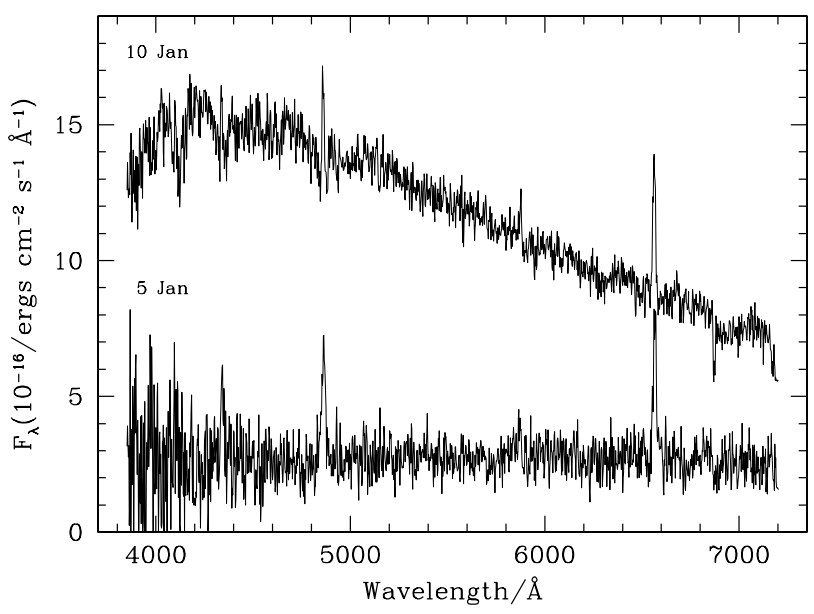

Fig. 2. Spectra of SY Vol. The first, taken on 5 January 2005, shows the system in quiescence. SY Vol was probably on the decay from outburst on 10 January 2005 , when the second spectrum was taken.

were made in white light; with the UCT CCD this gives photometry with an effective wavelength similar to Johnson $V$, but with a very broad bandpass. The non-standard flux distribution of CVs and the use of white light means that the high-speed observations cannot be precisely placed on a standard photometric system. The magnitude calibration to "pseudo- $V$ " approximates Johnson $V$ to within $\simeq 0.1 \mathrm{mag}$; colour terms were neglected in the atmospheric extinction corrections.

\subsection{SY Vol}

SY Vol is a little studied dwarf nova ranging in brightness from $V \simeq 14.4$ to $V \simeq 18.3$ (Woudt et al. 2005). Cieslinski et al. (1998) show a spectrum of SY Vol taken in outburst, while highspeed photometry in quiescence as well as outburst is presented by Woudt et al. (2005). The outburst recurrence timescale and orbital period of the system are not known.

Figure 2 displays our spectra of SY Vol. The first was taken when the system was in quiescence; it shows Balmer emission lines on a flat continuum. A second spectrum, taken five days later, shows SY Vol brighter, probably on the decline from outburst. This spectrum is very similar to the one presented by Cieslinski et al. (1998), showing a blue continuum and Balmer lines with broad absorption wings. He i $\lambda 5876$ emission is weakly detected in both spectra.

We took high-speed photometry of SY Vol on four occasions; these observations show the rapid flickering commonly seen in CVs. The systems also displayed a small systematic decline in brightness over the 4 nights for which we have photometry. This data set reveals no clear sign of an orbital modulation. We also searched all our light curves for periodic or quasiperiodic variations on shorter time scales, but found none. The light curves are displayed in Fig. 3, although they add little to

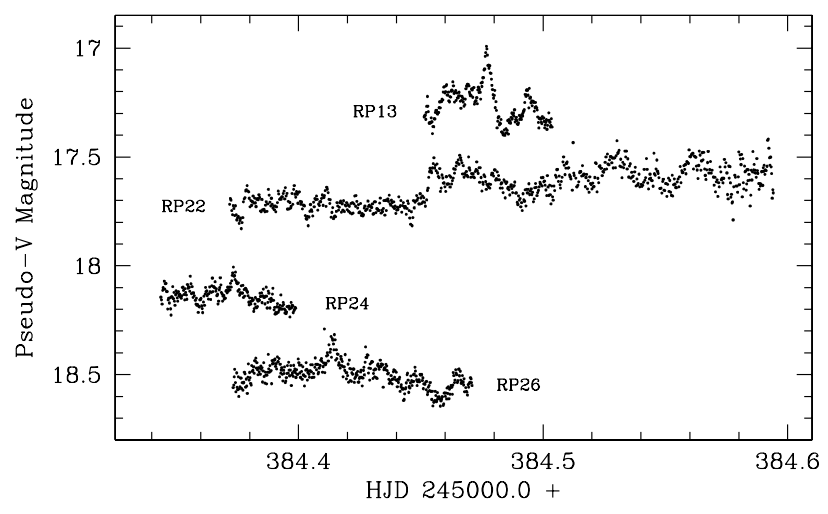

Fig. 3. The light curves of SY Vol. The run RP13, RP24, and RP26 observations are displaced vertically by $-0.35,+0.3$, and +0.6 mag respectively, and horizontal shifts of $-2 \mathrm{~d},-3 \mathrm{~d}$, and $-4 \mathrm{~d}$ respectively were applied to the RP22, RP24, and RP26 light curves.

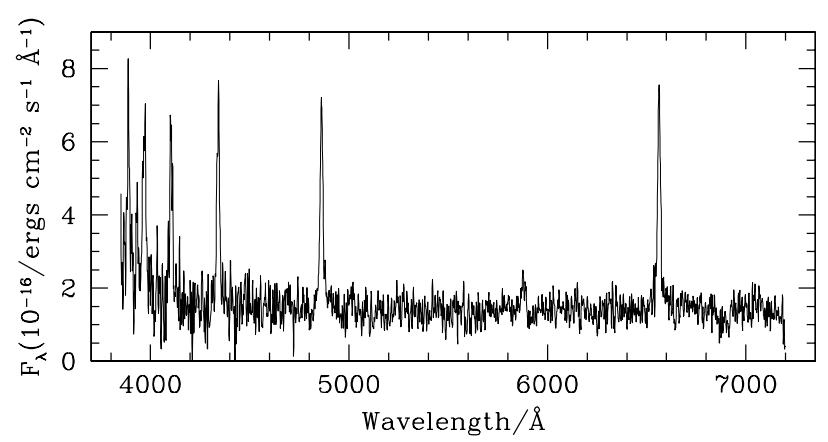

Fig. 4. The average of the two spectra of 1RXS J092737.4-191529. Broad Balmer and He I emission lines indicate that this object is a CV. The strength of the emission lines and flat continuum suggest that 1RXS J092737.4-191529 is a low- $\dot{M}$ system.

the study of Woudt et al. (2005). The low quality of the second half of run RP22 resulted from poor seeing.

The fact that all published studies of SY Vol include observations of high and low photometric states suggests that this system has a high DN outburst duty cycle.

\section{2. $1 R X S$ J092737.4-191529}

The optical counterpart of 1RXS J092737.4-191529 varied from $R=18.3$ to $R=15.4$ between the two epochs of photographic data. The system was faint (near 18th magnitude) during all our observations. There was no significant difference between the two spectra we took; the average of these two observations is displayed in Fig. 4. The spectrum has a flat continuum (longward of $\simeq 4500 \AA$ ) and Balmer as well as $\mathrm{He}_{\mathrm{I}} \lambda 5876$ emission lines. Both the strong emission lines and the flat continuum indicate that 1RXS J092737.4-191529 is a low- $\dot{M}$ system, probably a quiescent DN.

We obtained time-resolved photometry of 1RXS J092737.4191529 on four nights. The 14 January 2005 run was interrupted by cloud after less than an hour; the remaining three light curves are displayed in Fig. 5. The system flickers over a range of roughly $0.8 \mathrm{mag}$. Flickering is the hallmark of mass transfer, and its presence in the light curves confirms that 1RXS J092737.4191529 is a CV. The longest observation spanned more than $4 \mathrm{~h}$, but reveals nothing that is easily recognised as an orbital modulation. A Fourier analysis failed to reveal any coherent or quasicoherent modulations in the photometry. 
Table 3. Log of the time-resolved photometric observations. Dates are for the start of the night; HJD is for the middle of the first integration; the average magnitude for each run is listed in the final column.

\begin{tabular}{lllllll}
\hline \hline Object & Run no. & Date & HJD 2453000.0+ & Length/h & $t_{\text {int }} / \mathrm{s}$ & pseudo- $V$ \\
\hline SY Vol & RP13 & 13 Jan. 2005 & 384.45133 & 1.25 & 20 & 17.7 \\
& RP22 & 15 Jan. 2005 & 386.37182 & 5.42 & 20,26 & 17.9 \\
& RP24 & 16 Jan. 2005 & 387.34376 & 1.32 & 20 & 17.9 \\
& RP26 & 17 Jan. 2005 & 388.37330 & 2.35 & 20 & 18.1 \\
1RXS J092737.4-191529 & RP16 & 13 Jan. 2005 & 384.56121 & 3.40 & 24 & 18.1 \\
& RP20 & 14 Jan. 2005 & 385.45315 & 0.63 & 30 & $18.2:$ \\
& RP25 & 16 Jan. 2005 & 387.42975 & 4.37 & 20 & 18.2 \\
& RP27 & 17 Jan. 2005 & 388.50430 & 2.28 & 20 & 18.3 \\
\hline
\end{tabular}

Notes: $t_{\text {int }}$ is the integration time (the photometer is a frame transfer CCD, so that there is no dead time between exposures); ":" denotes an uncertain value.

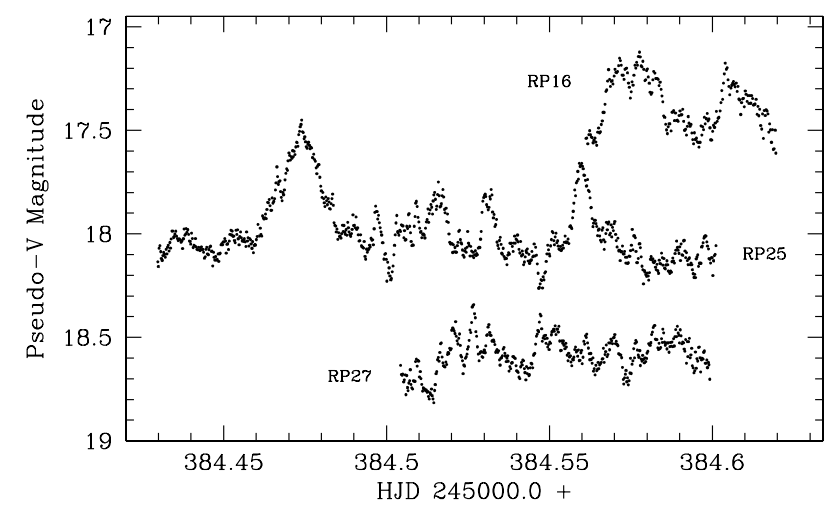

Fig. 5. Our 3 longer light curves of 1RXS J092737.4-191529, showing flickering amplitudes of up to $\simeq 0.4$ mag. The run RP27 light curve is shifted horizontally by $-4 \mathrm{~d}$ and vertically by $+0.5 \mathrm{mag}$. Run RP16 and RP25 are displaced by -0.5 mag and -3 d respectively.

\section{Discussion and summary}

Requiring ROSAT detection as well as at least 2 mag variability, we selected three objects for follow-up observations. The optical selection is based on a very incomplete sample, and we have used only two epochs of optical observations. Two of our three targets are $\mathrm{CVs}$, suggesting that this selection may be very efficient for finding CVs. Clearly, selection based on variability will be improved by higher quality optical data and/or more epochs. With large CCD mosaics becoming more common, wide-angle, multi-epoch surveys with much better photometric accuracy will soon be available. Relatively deep variability surveys currently in progress are aimed at short time scale variability, and cover smaller areas (e.g. Groot et al. 2003; Ramsay \& Hakala 2005). However, it will in future be possible to do all-sky surveys with $\sim 1 \mathrm{~d}$ sampling (Walker 2003). An obvious advantage that existing photographic archives will always have is their observational baseline of several decades.

In summary, we have presented observations of two CVs that were selected for large amplitude optical variability and X-ray emission. 1RXS J092737.4-191529 is a newly discovered system, while the optical counterpart of 1RXS J085325.4-711255 is the known DN, SY Vol. 1RXS J092737.4-191529 has the observational appearance of a low $-\dot{M}$ system, and is probably also a DN. This is the class of CVs that our selection is the most sensitive to.

Acknowledgements. M.L.P. acknowledges financial support from the South African National Research Foundation and the University of Southampton.

\section{References}

Bruch, A., \& Engel, A. 1994, A\&AS, 104, 79

Cieslinski, D., Steiner, J. E., \& Jablonski, F. J. 1998, A\&AS, 131, 119 Cropper, M. 1990, Space Sci. Rev., 54, 195

Gänsicke, B. T. 2005, in The Astrophysics of Cataclysmic Variables and Related Objects, ed. J.-M. Hameury, \& J.-P. Lasota, ASP Conf. Ser., 330, 3 Gänsicke, B. T., Marsh, T. R., Edge, A., et al. 2005, MNRAS, 361, 141 Groot, P. J., Vreeswijk, P. M., Huber, M. E., et al. 2003, MNRAS, 339, 427 Hambly, N., Read, M., Mann, R., et al. 2004, in Astronomical Data Analysis Software and Systems (ADASS) XIII, ed. F. Ochsenbein, M. G. Allen, \& D. Egret, ASP Conf. Ser., 314, 137

Hambly, N. C., Davenhall, A. C., Irwin, M. J., \& MacGillivray, H. T. 2001a, MNRAS, 326, 1315

Hambly, N. C., Irwin, M. J., \& MacGillivray, H. T. 2001b, MNRAS, 326, 1295 Hambly, N. C., MacGillivray, H. T., Read, M. A., et al. 2001c, MNRAS, 326, 1279

Hellier, C., Mukai, K., \& Beardmore, A. P. 1997, MNRAS, 292, 397

Kim, S.-W., Wheeler, J. C., \& Mineshige, S. 1992, ApJ, 384, 269

Livio, M., \& Pringle, J. E. 1994, ApJ, 427, 956

O’Donoghue, D. 1995, Baltic Astronomy, 4, 519

Osaki, Y. 1996, PASP, 108, 39

Patterson, J. 1994, PASP, 106, 209

Patterson, J., \& Raymond, J. C. 1985, ApJ, 292, 535

Ramsay, G., \& Hakala, P. 2005, MNRAS, 360, 314

Schwope, A. D., Brunner, H., Buckley, D., et al. 2002, A\&A, 396, 895 van Teeseling, A., Beuermann, K., \& Verbunt, F. 1996, A\&A, 315, 467 Verbunt, F., Bunk, W. H., Ritter, H., \& Pfeffermann, E. 1997, A\&A, 327, 602

Voges, W., Aschenbach, B., Boller, T., et al. 1999, A\&A, 349, 389

Voges, W., Aschenbach, B., Boller, T., et al. 2000, IAU Circ., 7432, 3

Wade, R. A., \& Ward, M. J. 1985, Cataclysmic variables: observational overview (Interacting Binary Stars, ed. J. E. Pringle, \& R. A. Wade, Cambridge Astrophysics Series (Cambridge: Cambridge University Press), 129

Walker, A. R. 2003, Mem. Soci. Astron. Ital., 74, 999

Warner, B. 1995, Cataclysmic variable stars (Cambridge Astrophysics Series, Cambridge, New York: Cambridge University Press)

Warner, B. 1996, Ap\&SS, 241, 263

Wheatley, P. J., Burleigh, M. R., \& Watson, M. G. 2000, MNRAS, 317, 343

Woudt, P. A., Warner, B., \& Spark, M. 2005, MNRAS, 364, 107 